\title{
Krisen, Nachhaltigkeit und das IASS
}

von Klaus Töpfer und Joachim Jens Hesse

Eine der aktuellen Situation gewidmete Positionsbestimmung verpflichtet in besonderer Weise dazu, die sich häufenden krisenhaften Erschütterungen und fundamentalen Verwerfungen zu analysieren, mit denen sich die Welt gegenwärtig konfrontiert sieht. Es gilt, Wirkungsmechanismen aufzudecken und daraus Hinweise darauf zu gewinnen, wie diese Krisen situativ bewältigt, problemorientiert abgearbeitet und schließlich auch ursächlich vermieden werden können. Aktuell steht fraglos die globale Finanz- und Wirtschaftskrise im Mittelpunkt politischer Entscheidungszwänge und gesellschaftlicher Besorgnisse. Die Eindämmung und Minimierung der dramatischen Konsequenzen spekulativer Verzerrungen im Nachgang systemgefährdender finanzmarktlicher Entscheidungen hat staatliche Maßnahmenpakete erzwungen, deren finanzielle Lasten zu einer nie für möglich gehaltenen Schuldenkrise der öffentlichen Hände in Deutschland, in Europa und weltweit führten. Besonders die Städte und die kommunalen Gebietskörperschaften verloren im Verlauf dieser Entwicklung weitgehend ihre Handlungsfähigkeit. Diese der Finanzkrise geschuldeten Verwerfungen der öffentlichen Haushalte brachte einige Länder der Eurozone, die ohnedies mit strukturellen Defiziten konfrontiert waren und diese oft nur mühsam verdecken konnten, in die konkrete Gefahr eines Staatsbankrotts. Die Mitgliedsländer der Eurozone sahen sich angesichts der durch massive Spekulationen gegen den Euro verstärkten Risiken verpflichtet, „Mauern“ gegen dieses Verhalten aufzubauen, wirtschaftliche Destabilisierungen und massiven Arbeitsplatzverlust zu vermeiden und eine die Existenz der gemeinsamen Währung bedrohende Krise zu bekämpfen.

Diese kurzfristig, geradezu hektisch zu treffenden Entscheidungen trugen jedoch dazu bei, dass der Blick von anderen Krisen abgelenkt wurde. Gleichwohl zählt auch die Umweltkrise, insbesondere die immer stärker durch Irreversibilitäten gekennzeichnete Klimakrise, zu den elementaren Herausforderungen an die politisch-administrativen Entscheidungsträger. Es zeichnet sich die große Gefahr $\mathrm{ab}$, dass der Hinweis auf die zwingende Bewältigung der Finanzkrise ein drin- 
gend erforderliches nationales, europäisches und globales Handeln gegen die Klimaveränderung in den Hintergrund treten lässt und so zum Verlust wertvoller Monate und Jahre im Ringen um ein nachhaltiges Klimaregime beiträgt. Diese Entwicklung wird zweifellos auch dadurch verstärkt, dass eine wieder ansteigende Zahl von Menschen weltweit die wissenschaftliche Basis des Klimawandels in Frage stellt oder doch vor einem entschlossenen Handeln die klimawissenschaftliche Grundlage zur weiteren Durchdringung an die Wissenschaft zurückverweisen möchte. Eine erhebliche zeitliche Verzögerung konzentrierten Handelns gegen den Klimawandel wurde zudem dadurch verstärkt, dass die Ergebnisse der Klimakonferenz in Kopenhagen allgemein als „unzureichend“ empfunden und entsprechend kommentiert wurden. Vielen ist dies ein willkommenes Alibi dafür, die Krise des Klimas bei der Bestimmung der Prioritäten politischen Handelns und gesellschaftlichen Bewusstseins deutlich nach hinten zu verlagern. Man wartet „gerne“ auf tragfähige Verhandlungsergebnisse von Diplomatie und Politik und sieht sich zwischenzeitlich nicht zum Handeln verpflichtet.

Vor diesem Hintergrund ist es in besonderer Weise erforderlich, die Ursachen beider Krisen aufzudecken und danach zu fragen, inwieweit sie in den wesentlichen Grundpositionen gleichen Mustern folgen. Die Umwelt- und Klimakrise ist entscheidend dadurch geprägt, dass umweltrelevante Kosten bei Produktion und Konsum aus den jeweiligen Kostenkalkulationen ausgeklammert und auf die Zukunft, auf Menschen in anderen Regionen oder auf die Assimilationskraft der Natur abgewälzt wurden. Umweltpolitik erweist sich auch in diesem Zusammenhang nicht als Kosten verursachend, umweltpolitische Entscheidungen erweisen sich vielmehr als Verteilungsentscheidungen. Umweltpolitik entscheidet mithin darüber, wer wann welche Kosten trägt. Die kurzfristigen Wohlstandskalkulationen erweisen sich aufgrund der benannte Abwälzung von Kosten als ökologisch - und sicherlich auch als sozial - hochgradig subventioniert. Die Klimaveränderungen sind somit Beleg dafür, dass die Assimilationskraft der großen Ökosysteme, vornehmlich der Atmosphäre, der Ozeane und der Biomasseproduktion, überreizt wurde. Aus der Kurzfristigkeit des Denkens bei der Berücksichtigung von Folgekosten ergibt sich ein turning point für die Stabilität dieser Ökosysteme. Insofern erweist sich die Umwelt- und Klimakrise als ein „Offenbarungseid der Kurzfristigkeit“.

Fragt man in gleicher Weise nach den begründenden Ursachen der Finanz- und Wirtschaftskrise, so zeigt sich, dass mit einer Fixierung auf Quartalsergebnisse und mit einer Trennung von Risikoübernahme und Haftung ein massiver Anreiz 
verbunden ist, Risiken und Kostengrößen wo immer möglich aus den kurzfristigen, für die Einkommensberechnung entscheidenden Kalkulationen herauszunehmen und sie auf externe Rechnungen zu übertragen oder gar gänzlich zu unterdrücken. Das „Diktat der Kurzfristigkeit“, unter dem die Wertbeurteilungen von Analysten erfolgen und das auch Bonusregelungen zugrunde liegt, hat entscheidend zu der dramatischen, spekulativen und unverantwortlichen Abkoppelung der Finanztransaktionen von der realen Wirtschaft beigetragen. Die Finanzund Wirtschaftskrise erweist sich somit ebenfalls als ein „Offenbarungseid der Kurzfristigkeit“.

Die grundsätzlich vergleichbaren Ursachen- und Wirkungsketten der beiden hier angesprochenen krisenhaften Erschütterungen und Verwerfungen führen zu der Frage, inwieweit in den Maßnahmepaketen zu der Bekämpfung der einen Krise, der Finanzkrise, nicht schon jene Maßnahmen verstärkt eingebunden werden müssten, die gleichzeitig bei der Bekämpfung der anderen Krise, der Umweltund Klimakrise, hilfreich und geboten wären. Die Überwindung des Diktats der Kurzfristigkeit, mithin die Einbindung der mittel- und langfristigen Konsequenzen aktuellen Handelns, sind die konstituierenden Parameter nachhaltigen Handelns, das weder die Lebens- und Entwicklungschancen der Menschen in anderen Regionen dieser Welt (und vor allem kommender Generationen) noch die Stabilität der Natur- und Ökosysteme in Frage stellt. Ein solcher Sinneswandel muss stets die Grundlage einer auf Nachhaltigkeit ausgerichteten Entwicklung sein.

Die notwendigen Grundlagen hierfür müssen zudem engagiert erforscht werden; es geht im Kern um die Frage, wie diese langfristigen Perspektiven in das tagespolitische Geschäft einer offenen demokratischen Gesellschaft eingebunden und langfristig mehrheitsfähig werden können. Es ist eine der wichtigen Aufgaben des Institutes for Advanced Sustainability Studies (IASS), das gegenwärtig in Potsdam aufgebaut wird, sich dieser Herausforderung zu stellen. Dies geht offensichtlich über die ohnedies zwingend erforderliche Interdisziplinarität hinaus und macht einen umfassenden Ansatz transdisziplinärer Arbeiten erforderlich. Ebenso wird es Aufgabe des Instituts sein, Spitzenforschern jenen Freiraum zu schaffen, den Sie zur Analyse von Lösungsvorschlägen für die langfristigen Problemstellungen gegenwärtiger Energiesysteme oder ökologischer Stressfaktoren benötigen. Dies gilt etwa für die Frage, inwieweit fossile Energieträger so genutzt werden, dass die bekannten klimatischen Folgen vermieden werden können, inwiefern $\mathrm{CO}_{2}$ als Rohstoff entwickelt werden kann und inwieweit der Kohlenstoffkreislauf als geschlossenes System zu konzipieren ist - Fragen von 
höchster Bedeutung in einer Welt, in der vor allem die Schwellenländern noch langfristig auf fossile Kohle als Energieträger angewiesen sein werden. Darin besteht eine wissenschaftliche Herausforderung, der sich der Physiknobelpreisträger Carlo Rubbia als Wissenschaftlicher Direktor im IASS besonders stellen wird. Andere Fragestellungen von weit reichender Bedeutung verbinden sich mit den Wirkungsmechanismen der Cryosphäre, vornehmlich in der Konzentration auf die Wirkung von Aeorosolen und „Black Carbon“-Partikeln.

Jenseits dieser natur- und technikwissenschaftlichen Fragestellungen muss sich die wissenschaftliche Spitzenforschung zum Thema „Nachhaltigkeit“ jedoch auch mit den notwendigen Voraussetzungen einer entsprechenden Ausrichtung des öffentlichen Handelns (staatlich wie überstaatlich) befassen. Im Zentrum steht hier die Frage nach der politisch-administrativen Auswahl und Koordination verfügbarer Maßnahmen, die als notwendige Bedingung für eine konzertierte und mithin effektive Antwort auf die benannten transnationalen Herausforderungen gelten darf. Hinausgehend über den heute allgemein bekannten Konflikt zwischen der territorial begrenzten Wirkungsmacht nationalstaatlichen Handelns und jenen materiellen Problemstellungen, die sich naturgemäß nicht an Staatsgrenzen orientieren, verbindet sich die Diskussion um die Kurskorrektur (oder -umkehr) in Richtung einer intelligenten und nachhaltigen wirtschaftlichen Entwicklung zunehmend mit grundlegenderen Fragen nach (a.) der gesellschaftlichen Wahrnehmung der Problemstellung sowie (b.) der Leistungs- und Reaktionsfähigkeit des bestehenden politisch-administrativen und institutionell-regulativen Rahmens. Im Kontext dieses Diskurses erscheint es von zentraler Bedeutung, zwischen dem Normativen und dem Funktionalen, dem Idealistischen und dem Pragmatischen, dem Wollen und dem Können, schließlich auch zwischen dem Illusionären und dem Machbaren zu unterscheiden, um die dringend angezeigte breite Erörterung nachhaltiger Entwicklung nicht durch selbstreferentielle Tendenzen ins Leere laufen zu lassen.

Angesichts weltweit erkennbarer Bemühungen um Deregulierung und Entnationalisierung, die verstärkt auch in komplexen Organisationsformen über Staatsgrenzen hinweg agierender Unternehmen und Interessengruppen ihren Ausdruck finden, ist zu erwarten, dass der Nationalstaat seine traditionell zentrale Rolle und Funktion bei der Erstellung und dem Vollzug verbindlicher Regelungssysteme zwar nicht verliert, aber schrittweise einbüßt (oder abgibt). Und in der Tat finden sich für bedeutende Teilaspekte moderner Gesellschaften heute zahlreiche transnationale Regelungsformen, vom Internet über die Welt des Sports bis hin zu Entwicklungspolitiken unterschiedlicher Intensität und Ausrichtung. Doch 
selbst in diesen Bereichen dürfte es unstrittig sein, dass dem staatlichen Handeln weiterhin eine beträchtliche Aufgabe zukommt, die sich unter Einschluss von Fragen der demokratischen Legitimation und Kontrolle nicht zuletzt auf die materielle Umsetzung und den Vollzug grenzüberschreitender Regelungsformen und nachfolgender Politiken richten. So verweisen nationalstaatliche Einrichtungen auf ein vielfältiges und vielfach erprobtes Instrumentarium, das im Kontext eines einheitlichen Rechtsrahmens und einer strukturierten Öffentlichkeit effektiv und problemnah die Leitlinien politischer Entscheidungen umzusetzen, mithin zu konkretisieren vermag. Darüber hinaus verbleiben nationalstaatliche Regierungen bekanntlich die Instanzen der Letztentscheidung im Rahmen der bestehenden, sich auf Nachhaltigkeitsfragen richtenden multilateralen Kooperations- und Koordinationsmechanismen. Entwicklungen hin zu arbeitsfähigen supranationalen Formen der Regelsetzung und -überwachung, wie etwa im Rahmen der Europäischen Union, blieben bislang die Ausnahme.

Eine Analyse der sich mit Nachhaltigkeitsfragen verbindenden Herausforderungen an das öffentliche (wie privatwirtschaftliche) Handeln wird sich im Rahmen der angesprochenen Wissenschaftsdisziplinen, also vor allem der Rechts-, Wirtschafts- und Sozialwissenschaften, auch auf absehbare Zeit an nationalstaatlichen Einrichtungen ausrichten und bei veränderten Verfahren eine staatsbezogene Perspektive wählen. Daraus ergibt sich mit Blick auf die jeweiligen institutionellen Kontexte und das Potential des Politisch-Administrativen eine wenigstens dreifache Differenzierung:

- „Klassische“ politisch-administrative Steuerung vermag verbindliche rechtliche und, darauf aufbauend, gesellschaftliche Rahmenbedingungen zu schaffen, wobei dies in unterschiedlicher Gewichtung für internationale, nationale, regionale und lokale Einheiten gilt.

- Durch Formen positiver wie negativer Anreizsetzung kann der politisch-administrative Bereich zudem Private dazu anhalten bzw. sie davon abbringen, bestimmte Handlungsmuster zu verfolgen und so zu einem aggregierten Resultat beizutragen.

- Schließlich kann der Staat als Teilnehmer an gesellschaftlich-ökonomischen Prozessen eine nicht unbedeutende Wirkung dadurch entfalten, dass er etwa über Ge- und Verbote, Zugangsbeschränkungen oder die Vorgabe von best practice-Modellen jene „Korridore“ entwickelt, innerhalb derer nachhaltige Entwicklung Fuß fassen müsste, um tatsächlich verstetigte Formen des individuellen wie kollektiven Verhaltens zu gewährleisten. 
Daraus folgt, dass sich der politisch-administrative Bereich insofern von anderen Akteursgruppen unterscheidet, als ihm in seiner einzigartigen Funktion bei der Schaffung und Aufrechterhaltung kollektiv verbindlicher Regelsysteme eine zentrale gestaltende Rolle zukommt, er aber bereits in Frühphasen der gesellschaftlichen Unterstützung und Legitimation bedarf, mithin auf ein verstärktes öffentliches Engagement angewiesen ist. Im Prozess der Umsetzung wiederum gewinnt die Interaktion mit Privaten, und hier vor allem dem privatwirtschaftlichen Bereich, an Bedeutung, kommen aber auch gesellschaftliche Belange, etwa über spezifische Zielgruppenorientierungen und die Wirkungen einzelner Steuerungsprozesse, wieder zur Geltung. Wenn Nachhaltigkeitsfragen also von einer Reihe unterschiedlicher Variablen gesellschaftlicher, kultureller, rechtlicher, ökonomischer und haushalterischer Natur beeinflusst werden, kanalisiert und katalysiert der politische Prozess den Einfluss dieser Variablen und vermag unter bestimmten Voraussetzungen und der Mitwirkung Dritter ein koordiniertes Ergebnis zu erwirken.

Die benannten Voraussetzungen zu analysieren, zu systematisieren, zu konkretisieren und in handlungsleitende Schlussfolgerungen zu überführen, sollte Aufgabe einer staats- und verwaltungswissenschaftlichen Analyse von Prozessen zur Gewährleistung von Nachhaltigkeit sein. Dabei wäre zwischen der hardware (Institutionen, Regelungssysteme, Kompetenzstrukturen, Instrumente) und der software (Anreize, Verfahren, Allokationsprozesse, Überzeugungsarbeit) des politisch-administrativen Handelns zu unterscheiden, um eine Differenzierung von Ursache und Wirkung, effect und by-product, intended und unintended outcomes, Aktion und Reaktion sowie Effektivität und Effizienz zu ermöglichen. Ziel ist es, die Vorbedingungen und Voraussetzungen sowie die Kosten und den Nutzen einer politisch-administrativen Nachhaltigkeitsstrategie zu erörtern und damit die benannten Fragestellungen (wenigstens in Teilen) zu beantworten. Dem dient nicht zuletzt die in Aussicht genommene Kooperation des IASS mit dem Internationalen Institut für Staats- und Europawissenschaften (ISE) in Berlin.

Das IASS wird darüber hinaus gesamthaft bestrebt sein müssen, immer wieder jene wissenschaftlichen Herausforderungen anzunehmen, die unter dem Gesichtspunkt eines dem „Diktat der Kurzfristigkeit“" verpflichteten Wirtschafts-, Regierungs- und Gesellschaftssystems vernachlässigt werden und damit ein hohes Risikopotential für Stabilität und friedliches Zusammenleben in einer Welt mit bald neun Milliarden Menschen darstellen. So erwähnt der Bericht des IPCC die nicht auszuschließende Möglichkeit eines akuten climate emergency: Welche 
institutionellen und rechtlichen Strukturen müssen angesichts dieser Möglichkeit vorsorglich gedacht werden? Welche Konsequenzen wären damit verbunden, wenn sich regionale Entscheidungsträger für Maßnahmen eines climate engineering entscheiden sollten? Welche Wirkung entfalten kulturanthropoligische Unterschiede in dieser Welt mit Blick auf das Verständnis von Fortschritt und Nachhaltigkeit? Nachhaltigkeitsforschung lässt sich erkennbar nicht auf eine Fachdisziplin oder ein bestimmtes Forschungsprogramm reduzieren. So bleibt zu hoffen, dass sich das IASS als eine sinnvolle Ergänzung der in Deutschland bereits bestehenden Wissenschaftslandschaft erweist - nicht zuletzt auch im Bereich der Staatswissenschaften. 